\title{
Rituximab, bendamustine, and low-dose cytarabine as induction therapy in elderly patients with mantle cell lymphoma: a multicentre, phase 2 trial from Fondazione Italiana Linfomi
}

\author{
Carlo Visco, Annalisa Chiappella, Luca Nassi, Caterina Patti, Simone Ferrero, Daniela Barbero, Andrea Evangelista, Michele Spina, \\ Annalia Molinari, Luigi Rigacci, Monica Tani, Alice Di Rocco, Graziella Pinotti, Alberto Fabbri, Renato Zambello, Silvia Finotto, Manuel Gotti, \\ Angelo M Carella, Flavia Salvi, Stefano A Pileri, Marco Ladetto, Giovannino Ciccone, Gianluca Gaidano, Marco Ruggeri, Maurizio Martelli, \\ Umberto Vitolo
}

\section{Summary}

Background The combination of rituximab, bendamustine, and cytarabine (R-BAC) was highly active in a pilot trial of mantle cell lymphoma, but its use was restricted by high haematological toxicity. We aimed to assess the efficacy and safety of an R-BAC regimen with low-dose cytarabine (RBAC500).

Methods In this multicentre, phase 2 trial, we recruited previously untreated patients with an established histological diagnosis of mantle cell lymphoma from 29 Fondazione Italiana Linfomi centres in Italy. Patients had to be older than 65 years and fit according to the comprehensive geriatric assessment, or aged 60-65 years if they were ineligible for high-dose chemotherapy plus autologous stem-cell transplantation and were fit or unfit. All patients received RBAC500 (rituximab $375 \mathrm{mg} / \mathrm{m}^{2}$ on day 1, bendamustine $70 \mathrm{mg} / \mathrm{m}^{2}$ on days 2 and 3, and cytarabine $500 \mathrm{mg} / \mathrm{m}^{2}$ on days 2-4; all administered intravenously) every 4 weeks for up to six cycles. Primary endpoints were the proportion of patients achieving complete response at the end of treatment and toxicity, defined as the occurrence of any of the stop treatment criteria or of any episode of relevant toxicity. All patients who started at least one cycle of RBAC500 were included in the primary and safety analyses. Using efficacy and toxicity as a composite primary endpoint, we considered the final conclusion positive if more than 28 of 57 patients achieve complete response and fewer than 18 of 57 patients report toxicities. This study is registered with EudraCT, number 2011-005739-23, and ClinicalTrials.gov, number NCT01662050, and is completed.

Findings Between May 2, 2012, and Feb 25, 2014, we enrolled 57 patients (median age 71 years, IQR 67-75). 54 (95\%) patients received at least four RBAC500 cycles (three discontinued because of toxicity), and 38 (67\%) completed six cycles. Two (4\%) had disease progression (one after the fourth cycle and one after the sixth cycle). All 52 (91\%, lower limit of one-sided $95 \%$ CI $85 \%$ ) remaining patients achieved complete response at the end of treatment. 23 (40\%, upper limit of one-sided 95\% CI 53\%) of 57 patients had at least one episode of relevant toxicity. The most frequent grade 3-4 haematological toxicities were neutropenia (149 [49\%] of 304 cycles) and thrombocytopenia (158 [52\%]). Most treatment-related non-haematological adverse events were of grade 1-2, with the most frequent ones being fatigue (14 [25\%] patients), nausea or vomiting (12 [21\%]), and infusion-related reactions or tumour lysis syndrome (12 [21\%]). 41 (72\%) patients required a dose reduction. 12 patients died during the study, but no deaths were related to treatment.

Interpretation RBAC500 is an effective treatment for elderly patients with mantle cell lymphoma and, despite not meeting our prespecified safety boundary, haematological toxicity was manageable with appropriate supportive care and dose reduction. Since maintenance therapy is not required, RBAC500 could be considered an option and should be studied in phase 3 trials.

Funding Fondazione Italiana Linfomi and Mundipharma.

\section{Introduction}

Mantle cell lymphoma is an aggressive form of nonHodgkin lymphoma characterised by continuous relapses and no standard initial therapy. ${ }^{1}$ Effective, well tolerated, and affordable front-line therapeutic options are urgently needed. ${ }^{2}$ Traditional chemotherapy regimens such as R-CHOP (rituximab plus cyclophosphamide, doxorubicin, vincristine, and prednisone) have been associated with only modest complete response (34-48\%), with median progression-free survival of 16.6-22.1 months. ${ }^{3-5}$ The effectiveness of these regimens can be improved either by rituximab maintenance ${ }^{6}$ or by the replacement of vincristine with bortezomib. Additionally, bendamustine combined with rituximab has improved complete response and progression-free suvival compared with R-CHOP in two randomised

\section{(1)}

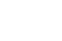


Oncology 1, Veneto Institute of Oncology-IRCCS, Padova, Italy (S Finotto MD); Hematology Oncology, Fondazione IRCCS Policlinico San Matteo, Pavia, Italy (M Gotti MD); past-director hematology unit IRCCS San Martino, Genova, Italy (A M Carella MD); Hematology, SS Antonio e Biagio e Cesare Arrigo Hospital, Alessandria, Italy (F Salvi MD, M Ladetto MD);

Haematopathology Unit, European Institute of Oncology, Milan, Italy (Prof S A Pileri MD); and Bologna University School of

Medicine, Bologna, Italy (Prof S A Pileri)

Correspondence to: Dr Carlo Visco, Department of Cell Therapy and Hematology,

San Bortolo Hospital, Via Rodolfi 37, 36100 Vicenza,

\section{Research in context}

\section{Evidence before this study}

To establish the best available upfront treatment for patients with mantle cell lymphoma who are ineligible for autologous stem-cell transplantation, we searched PubMed from Jan 1, 2010, to Sept 2, 2016, for all phase 2 and 3 trials, with no other inclusion criteria or restrictions, using the search terms "mantle cell lymphoma" and "elderly". We identified four large randomised studies. The first two studies showed that the efficacy of R-CHOP (rituximab plus

cyclophosphamide, doxorubicin, vincristine, and prednisone) can be significantly improved either by the addition of rituximab maintenance or by replacement of vincristine with bortezomib. The other two studies showed superiority of the bendamustine-rituximab regimen over $\mathrm{R}$-CHOP-like treatment. Moreover, a growing amount of data indicate that new targeted drugs are changing the landscape of mantle cell lymphoma therapy, with encouraging responses when administered alone or in combination with chemotherapy. $\mathrm{R}-\mathrm{BAC}$ is a regimen combining bendamustine and rituximab with intermediate-dose cytarabine, a drug that is considered the mainstay for treatment of younger patients (aged $<65$ years) with mantle cell lymphoma. In a pilot study, R-BAC has shown encouraging efficacy but substantial haematological toxicity.

\section{Added value of this study}

In this phase 2 study of R-BAC in the upfront treatment of elderly patients with mantle cell lymphoma, the dose of cytarabine was lowered to $500 \mathrm{mg} / \mathrm{m}^{2}$ (RBAC500).

Haematological toxicity was frequent but manageable with supportive care and dose reduction, and a high proportion of patients had a complete response, including durable responses of at least 3 years for most patients. Our results validate earlier findings and will hopefully contribute to research progress in an area where effective regimens are urgently needed.

\section{Implications of all the available evidence}

RBAC500 is an effacacious, tolerated regimen for elderly patients with mantle cell lymphoma. Its efficacy compares favourably with other regimens, with or without ritiximab maintenance, in this patient population. At present, expensive new drugs and combination regimens for mantle cell lymphoma are increasingly followed by prolonged maintenance. Results of this trial suggest that RBAC 500 should be tested in phase 3 studies, and could be considered as an inexpensive and short-term option. trials ${ }^{5,8}$ of mantle cell lymphoma and was well tolerated in older or frail patients.

The incorporation of high-dose cytarabine $\left(\geq 1 \mathrm{~g} / \mathrm{m}^{2}\right)$ has been widely recognised as highly beneficial in the treatment of mantle cell lymphoma, primarily in intensive regimens for younger patients (aged $<65$ years). ${ }^{9-11}$ Results from preclinical studies ${ }^{12,13}$ have shown that bendamustine and cytarabine have distinct and synergistic mechanisms of action in mantle cell lymphoma cell lines, especially when administered sequentially. The R-BAC (rituximab, bendamustine, and intermediate-dose cytarabine $\left[800 \mathrm{mg} / \mathrm{m}^{2}\right]$ ) regimen led to a $95 \%$ complete response in a preliminary pilot series of 20 previously untreated elderly patients (aged $\geq 65$ years) with mantle cell lymphoma. ${ }^{14}$ However, this regimen was associated with substantial haematological toxicity, especially grade 3-4 thrombocytopenia (70 [70\%] of 100 cycles).

In this trial, we aimed to assess whether reduction of cytarabine dose to $500 \mathrm{mg} / \mathrm{m}^{2}$ in the R-BAC regimen could decrease haematological toxicity while preserving antitumour activity in elderly patients with mantle cell lymphoma.

\section{Methods}

\section{Study design and participants}

In this multicentre, single-group, phase 2 study, we recruited previously untreated patients with an established histological diagnosis of mantle cell lymphoma on lymph node biopsy, bone marrow biopsy, or extranodal tissue from 29 Fondazione Italiana Linfomi centres in Italy. Patients were older than
65 years and fit according to the comprehensive geriatric assessment, or aged 60-65 years if they were ineligible for high-dose chemotherapy plus autologous stem-cell transplantation and were fit or unfit according to the comprehensive geriatric assessment (see appendix p 2 for the modified comprehensive geriatric assessment). The diagnostic criteria of mantle cell lymphoma included positivity for cyclin D1 and SOX11 expression (mandatory in patients who were cyclin D1 expression or $\mathrm{t}(11 ; 14)$ negative), and expression of CD20 and CD5. Eligible patients also had to have bidimensionally measurable disease, Eastern Cooperative Oncology Group (ECOG) performance status 0-2, and adequate cardiac function, renal function (creatinine clearance $>40 \mathrm{~mL} / \mathrm{min}$ ) with preserved diuresis, and hepatic function (alanine aminotransferase and aspartate aminotransferase $<2 \cdot 5$ times the upper limit of normal and total bilirubin $<2 \mathrm{mg} / \mathrm{dL}$, unless directly attributable to the patient's tumour). Any blood cell count was allowed, if it was related to bone marrow involvement.

Patients were excluded if they had in-situ mantle cell lymphoma; non-nodal leukaemic disease; active malignant diseases other than lymphoma; active hepatitis B or C; HIV positivity; medical conditions or organ injuries that could interfere with therapy administration; active bacterial, viral, or fungal infection requiring systemic therapy; seizure disorders requiring anticonvulsant therapy; severe chronic obstructive pulmonary disease with hypoxaemia; history of severe cardiac disease (New York Heart Association functional 
class III-IV, myocardial infarction within 6 months, ventricular tachyarrhythmias, dilatative cardiomyopathy, or unstable angina); uncontrolled diabetes; known hypersensitivity or anaphylactic reactions to murine antibodies and proteins, bendamustine, or mannitol; major surgery within 4 weeks of study day 1 ; CNS involvement; or any coexisting medical or psychological condition that would preclude participation in the study or compromise the patient's ability to give informed consent, or that might affect the interpretation of the results, or render the patient at high risk from treatment complications.

The study was done in accordance with the principles of the Declaration of Helsinki and Good Clinical Practice. Ethics approval was granted by the institutional review board at each participating institution, and all patients provided written informed consent.

\section{Procedures}

Baseline assessment included bone marrow biopsy, tumour staging with contrast-enhanced CT, and PET. Paraffin blocks of the diagnostic specimen were collected for central pathological review and central Ki67 assessment, which were done by an investigator (SAP) according to criteria of the WHO Classification (2008 edition). ${ }^{15}$

All patients received RBAC500 (rituximab $375 \mathrm{mg} / \mathrm{m}^{2}$ on day 1; bendamustine $70 \mathrm{mg} / \mathrm{m}^{2}$ over $30-60 \mathrm{~min}$ on days 2 and 3; cytarabine $500 \mathrm{mg} / \mathrm{m}^{2}$ over a $2 \mathrm{~h}$ infusion starting $2 \mathrm{~h}$ after bendamustine on days 2-4; all administered intravenously) every 4 weeks for up to six cycles. Prophylaxis with granulocyte colony-stimulating factor $(10 \mu \mathrm{g} / \mathrm{kg})$ was given 3 days after the last cytarabine dose in every cycle. Recovery of absolute neutrophil count to 1000 cells per $\mu \mathrm{L}$ and platelet count to 75000 platelets per $\mu \mathrm{L}$ was required before starting cycles. If grade 3-4 haematological toxicity was present on day 1 of the next cycle, subsequent cycles were postponed for a maximum of 2 weeks, and doses of bendamustine and cytarabine reduced by $25 \%$. Allopurinol (100-300 mg per day) was used for prevention of tumour lysis syndrome for at least the first 2 weeks of treatment. Prophylaxis with trimethoprim-sulfamethoxazole or pentacarinat aerosol was mandatory in all patients. Aciclovir was mandatory only in patients with a previous history of viral herpetic reactivations. The use of steroid-containing eyedrops was allowed but was restricted to the days of active treatment with cytarabine.

Patients who did not respond to the first two cycles were discontinued from the study. Response assessment after completion of therapy included CT, PET, and bone marrow examination. Patients subsequently had clinical examination, laboratory tests (blood counts, renal and hepatic function exams, lactate dehydrogenase), and CT scan every 3 months for the first year after end of treatment and then every 6 months for 2 more years (minimum 24 months of follow-up).
Centralised assessment of minimal residual disease was done at the University of Torino (Torino, Italy) at diagnosis, before cycle 3, and at 1 month, 6 months, 12 months, and 24 months after the end of treatment. We used allelespecific oligonucleotide nested PCR to assess minimal residual disease in bone marrow and peripheral blood samples, using patient-specific immunoglobulin heavychain variable region gene rearrangement or the BCL1 product of the $t(11 ; 14)$ translocation, or both (if available), as previously reported. ${ }^{16}$ The sensitivity of the nested PCR approach was three neoplastic rearrangements in $10^{6}$ normal cells (ie, $3.00 \times 10^{-6}$ ) in repeated dilution

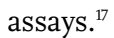

\section{Outcomes}

The primary efficacy outcome was the proportion of patients achieving complete response-based on results from CT, PET, and bone marrow biopsy and defined according to 2007 International Working Group response criteria — at the end of treatment. ${ }^{18}$ The primary safety outcome was toxicity, defined as the occurrence of any of the stop treatment criteria or of any episode of relevant toxicity. Stop treatment criteria included the occurrence of relevant toxicity for two subsequent or consecutive cycles, despite the $25 \%$ dose reduction, or grade 3-4 haematological or non-haematological toxicity on day 28 of a cycle that did not resolve within 2 weeks. Relevant toxicity was defined as grade 4 cytopenia lasting for more than 6 days, grade 3-4 non-haematological toxicity, or febrile neutropenia lasting for more than 3 consecutive days regardless of response to treatment.

Secondary outcomes were molecular response (ie, the proportion of patients with molecular rearrangements at baseline that became negative during treatment, measured by qualitative and quantitative PCR); progression-free survival from enrolment until disease progression, relapse, or death from any cause; overall survival from enrolment until death from any cause; duration of response (measured from the first assessment that documented complete or partial response to the date of disease relapse or progression); the proportion of patients completing all six RBAC500 cycles; and the proportion of patients who had dose reductions or delays.

Safety was assessed by documentation of adverse events (according to the US National Cancer Institute Common Terminology Criteria version 4.0), clinical laboratory results, vital signs, and physical examinations.

\section{Statistical analysis}

We adopted the Bryant and Day two-stage design ${ }^{19}$ for sample size calculation, with a composite efficacy-toxicity primary endpoint on the basis of our previous experience with R-BAC. ${ }^{14}$ The Bryant and Day design assumes that toxicity and activity are independent; although this assumption might be unlikely in some trials, the design has proved robust to mis-specification of the relation between toxicity and activity. ${ }^{20}$ We set the upper bounds 


\begin{tabular}{|c|c|}
\hline & Data $(n=57)$ \\
\hline Median age (IQR), years & $71(67-75)$ \\
\hline \multicolumn{2}{|l|}{ Sex } \\
\hline Male & $43(75 \%)$ \\
\hline Female & $14(25 \%)$ \\
\hline \multicolumn{2}{|l|}{ Ann Arbor stage } \\
\hline II & $5(9 \%)$ \\
\hline IIIIIV & $52(91 \%)$ \\
\hline \multicolumn{2}{|c|}{ Bone marrow involvement } \\
\hline Yes & $36(63 \%)$ \\
\hline No & $21(37 \%)$ \\
\hline \multicolumn{2}{|c|}{ Eastern Cooperative Oncology Group performance status } \\
\hline $0-1$ & $54(95 \%)$ \\
\hline 2 & $3(5 \%)$ \\
\hline \multicolumn{2}{|l|}{ Morphological variants } \\
\hline Classical & $43(75 \%)$ \\
\hline Pleomorphic & $8(14 \%)$ \\
\hline Blastoid & $6(11 \%)$ \\
\hline \multicolumn{2}{|l|}{ Ki67 index* } \\
\hline Median (IQR) & $20 \%(8-33)$ \\
\hline$<30 \%$ & $35 / 51(69 \%)$ \\
\hline$\geq 30 \%$ & $16 / 51(31 \%)$ \\
\hline \multicolumn{2}{|l|}{ MIPI } \\
\hline Low risk & $9(16 \%)$ \\
\hline Intermediate risk & $23(40 \%)$ \\
\hline High risk & $25(44 \%)$ \\
\hline \multicolumn{2}{|c|}{$\begin{array}{l}\text { Data are } \mathrm{n}(\%) \text { or } \mathrm{n} / \mathrm{N}(\%) \text { unless indicated otherwise. MIPI=Mantle Cell } \\
\text { Lymphoma International Prognostic Index. }{ }^{*} \text { Six patients had no Ki67 data since } \\
\text { they did not have available material for central revision. }\end{array}$} \\
\hline
\end{tabular}

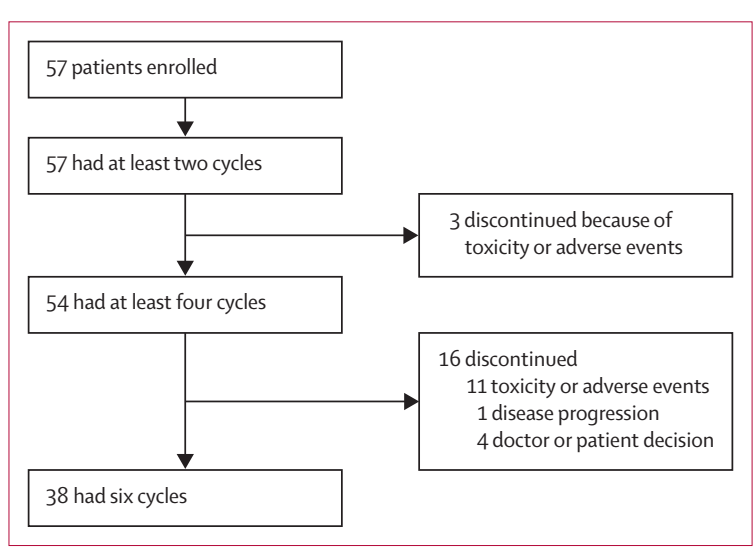

Figure 1: Trial profile

on the probabilities of erroneously recommending the treatment at 0.05 both when complete response was inadequate and when toxicity was unacceptable. The upper bound on the probability of failing to recommend the treatment when it was acceptable with respect to both response and toxicity was $0 \cdot 2$ ( $80 \%$ power). We fixed the probability of unacceptable complete response at
0.40 and the probability of acceptable complete response at 0.60 . In an analogous manner, the cutoffs for toxicity probabilities were 0.40 (unacceptable) and $0 \cdot 20$ (acceptable). According to these parameters, 19 patients were required for the first stage and 57 patients for the second stage. The final conclusion would be positive if more than 28 of 57 patients achieve complete response and fewer than 18 of 57 patients report toxicities. Therefore, we aimed to recruit 57 patients.

All patients who started at least one cycle of RBAC500 were included in the efficacy and safety analyses. We used descriptive statistics and their 95\% CIs to summarise the efficacy and safety endpoints. We reported only one-sided CIs since the Bryant and Day design implies that two one-sided hypotheses are to be tested (ie, proportion of responses larger than the null hypothesis and frequency of toxicities lower than the null hypothesis). Time-to-event variables were analysed with the Kaplan-Meier method. We used StatView (Abacus Concepts, Berkeley, CA, USA) for statistical analyses, and the data cutoff date for the final analysis was Aug 15, 2016. A data monitoring committee, composed of independent representatives of Fondazione Italiana Linfomi (pharmacovigilance office), oversaw the study. Pharmacovigilance and monitoring were done during study accrual and treatment phase. All serious adverse events and suspected unexpected serious adverse reactions were analysed in real time by an investigator (CV) and by an independent monitoring team of Fondazione Italiana Linfomi. This study was registered with EUDRA-CT, number 2011-005739-23, and ClinicalTrials.gov, number NCT01662050.

\section{Role of the funding source}

Fondazione Italiana Linfomi was involved in study design, data collection, data analysis, data interpretation, and writing of the report. Bendamustine was provided by Mundipharma Pharmaceuticals, which also provided a grant to Fondazione Italiana Linfomi to partly cover the management costs of this study; Mundipharma Pharmaceuticals had no role in study design, data collection, data analysis, data interpretation, or writing of the report, and did not see the final results before submission. The corresponding author had full access to all the data in the study and had final responsibility for the decision to submit for publication.

\section{Results}

Between May 2, 2012, and Feb 25, 2014, we enrolled 57 patients from 29 centres. 49 (86\%) patients had diagnostic biopsy samples confirmed by central pathology review as mantle cell lymophoma, and the remaining eight $(14 \%)$ patients were included after review of centrally submitted biopsy reports. No patient was excluded on the basis of central pathology review. Median age was 71 years (IQR 67-75, range 61-79), $43(75 \%)$ patients were male, and none presented with 
non-nodal leukaemic disease, in agreement with our inclusion criteria (table 1).

304 cycles of RBAC500 were given. Of 57 eligible patients, 54 (95\%) received at least four cycles of RBAC500, and 38 (67\%) had six cycles (figure 1), with a median of $6 \cdot 0$ (IQR 5.4-6.6, range 2-6) cycles per patient. Adverse events and toxicities leading to treatment discontinuation were febrile neutropenia $(n=1)$, documented infections (one patient had infection of the surgical wound from lymphoma diagnosis and one had persistent cytomegalovirus reactivation), haematological toxicity exceeding our previously defined criteria $(n=9)$, myocardial infarction with cerebral ischaemia $(n=1)$, and atrial fibrillation $(n=1)$. The most common haematological toxicities requiring discontinuation were thrombocytopenia $(n=3)$, leucopenia $(n=2)$, or both $(n=4)$.

Of 57 patients, $41(72 \%)$ had at least one dose reduction during the study. 30 patients (53\%) delayed the subsequent cycle at least once. As specified in the protocol, each delay lasted for fewer than 14 days (median 7 days, IQR 5-9). Overall, 14 (25\%) patients received the full-dose chemotherapy without dose reduction or delay. Granulocyte colony-stimulating factor was given to all patients.

Two (4\%) patients had disease progression, one after the fourth cycle and one after the sixth cycle. Three (5\%) patients received fewer than four cycles because of toxicity and were considered non-responders (figure 1). All 52 responding patients achieved complete response at the end of treatment (complete response 91\%, lower limit of one-sided $95 \%$ CI $85 \%$ ).

After a median follow-up of 35 months (IQR 30-41, range 28-52), 42 (74\%) patients were alive and disease free. Median progression-free survival was not reached (95\% CI 42-not reached), nor was median duration of response reached (54-not reached). 12 patients died. At 2 years, overall survival was $86 \%$ (74-93), progressionfree survival was $81 \%$ (68-89), and duration of response was $90 \%$ (85-94; figure 2). Progression-free survival was $76 \%(65-85)$ at median follow-up of 35 months. In posthoc subgroup analyses, adverse predictive factors affecting progression-free survival were high Mantle Cell Lymphoma International Prognostic Index (MIPI), elevated Ki67 ( $\geq 30 \%$ ), and blastoid morphology (figure 3; appendix p 2). In multivariate analysis, elevated Ki67 (hazard ratio $7.43,95 \%$ CI $1.75-31.57 ; \mathrm{p}=0.0062$ ) and blastoid variant $(4 \cdot 16,1 \cdot 12-18.52 ; \mathrm{p}=0.043)$ were independent predictors of worse progression-free survival (appendix p 3). Patients with either of these two features had a significantly worse 2 year progression-free survival (41\%, 19-63) compared with patients with classical or pleomorphic variants and low Ki67 (98\%, 84-100; figure 3D).

Of 57 patients, $45(79 \%)$ had a molecular marker (29 in the immunoglobulin heavy-chain variable region, eight had BCL1-based targets, and eight had both). No difference in clinical or pathological characteristics

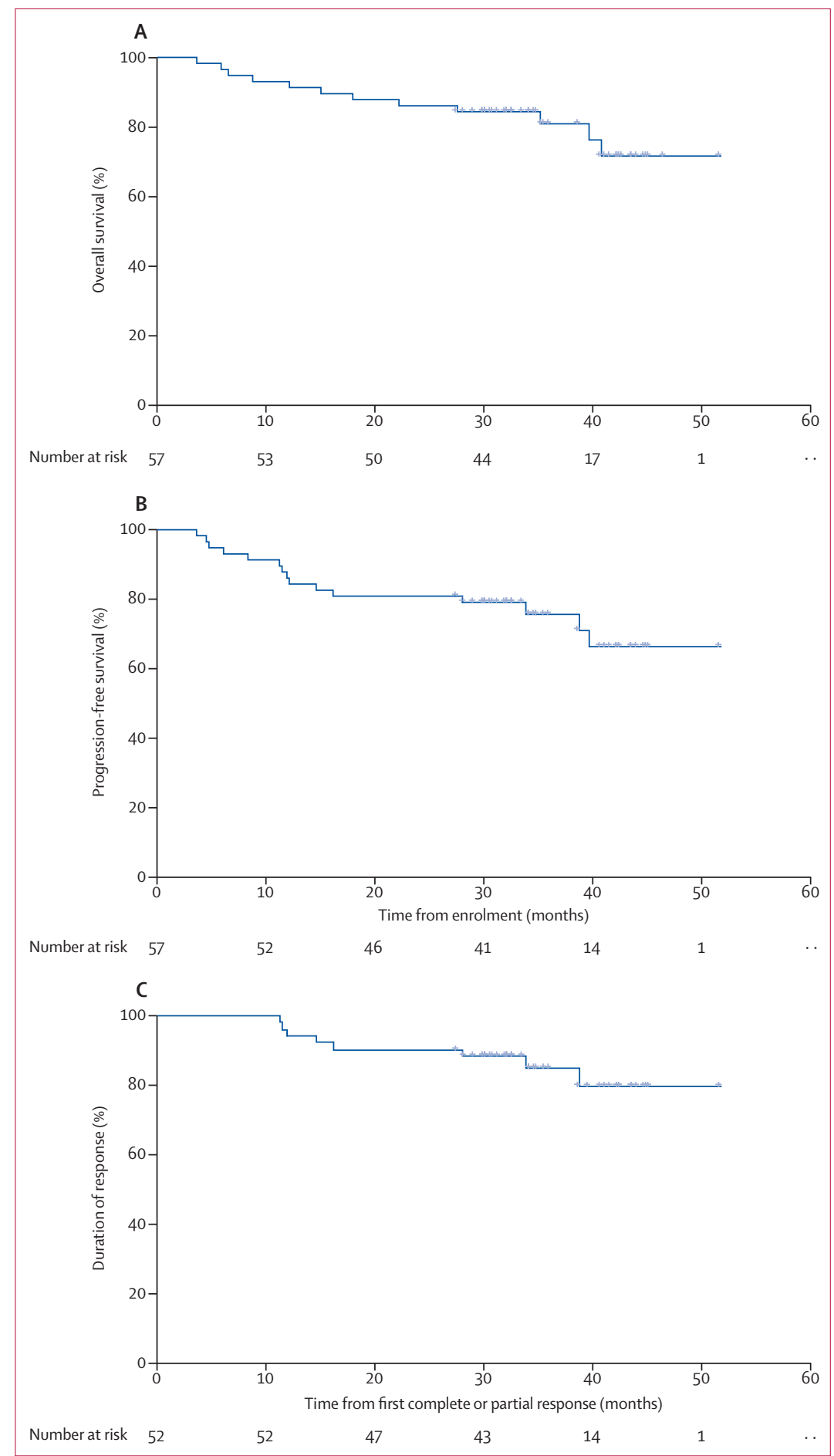

Figure 2: Kaplan-Meier plots

(A) Overall survival. (B) Progression-free survival. (C) Duration of response.

(demographics, Ki67, morphology, or MIPI) was seen between patients with a molecular marker and those without (appendix p 4). After two cycles of RBAC500, $24(54 \%)$ patients had molecular response in the bone 


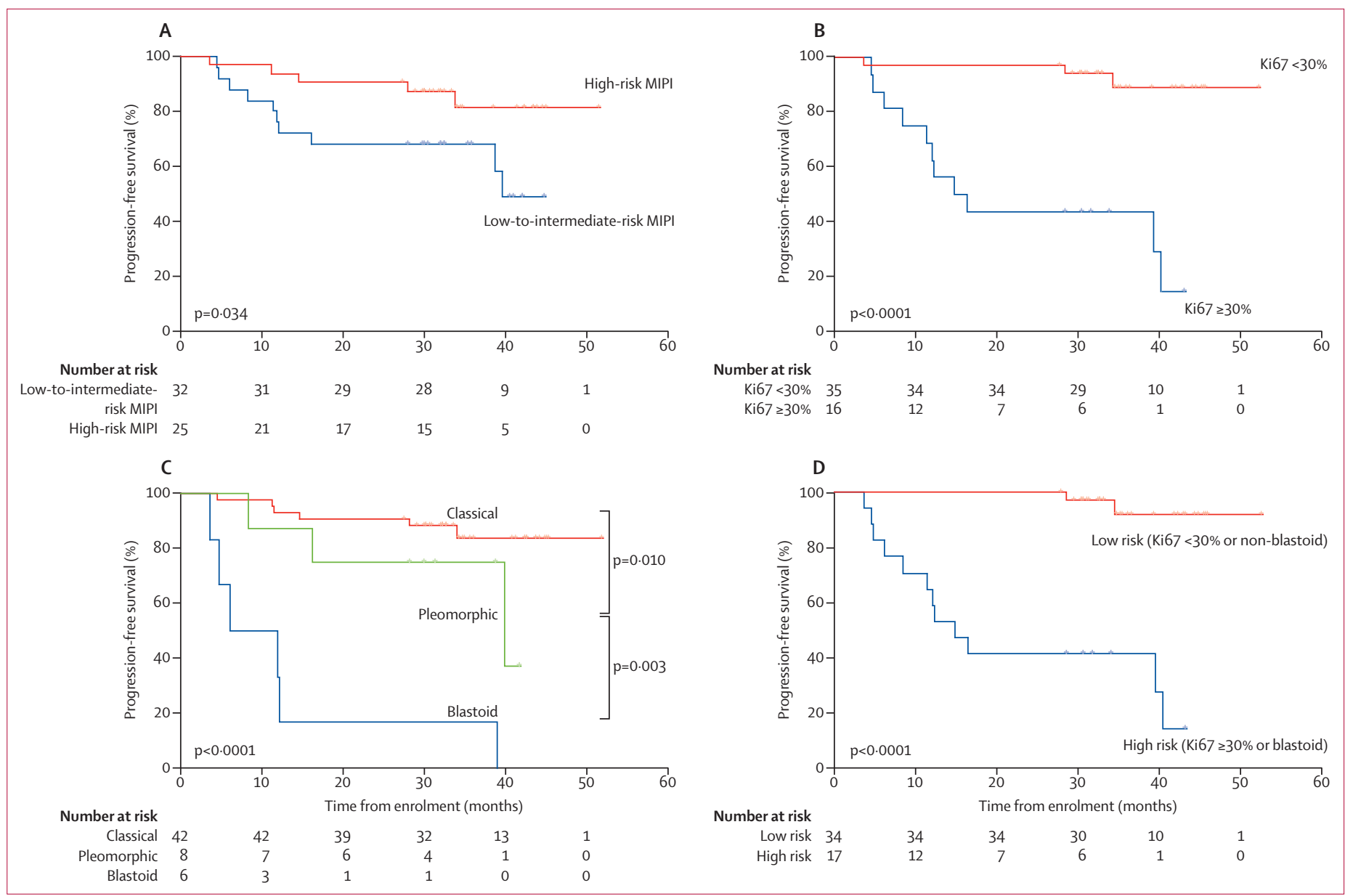

Figure 3: Subgroup analyses of progression-free survival

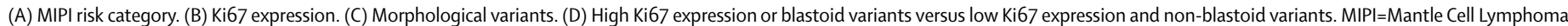
International Prognostic Index.

marrow and 28 (62\%) had molecular response in peripheral blood; at the end of treatment, 24 (54\%) had molecular response in the bone marrow and 35 (78\%) had molecular response in peripheral blood. Of the 28 patients with available sample 1 year after the end of treatment, 16 (57\%) had molecular response in the bone marrow and 21 (75\%) had molecular response in peripheral blood. In a post-hoc analysis, no association was found between molecular response and progressionfree survival, possibly because of the low number of events in this study. A comprehensive report on the association between molecular response and outcome, together with quantitative PCR findings, will be published separately.

The primary toxicity was reversible myelosuppression (table 2). Platelet transfusions were administered in $89(29 \%)$ of 304 delivered cycles. Overall, 23 (40\%, upper limit of one-sided $95 \%$ CI 53\%) of 57 patients had at least one episode of relevant toxicity. Most treatment-related non-haematological adverse events and common events were of grade 1-2. Unlike a previous study of R-BAC, ${ }^{14}$ elevation of $\gamma$-glutamyl transferase was rare with RBAC500. Six (11\%) patients had grade 3-4 documented infections or viral reactivations, and other grade 3-4 non-haematological toxicities included infusion-related reactions, fatigue, elevated glutamic-pyruvic transaminase and glutamicoxaloacetic transaminase, and cardiac events (table 3 ). No renal toxicity or grade 3-4 alopecia was reported. Of 41 patients who had dose reduction, 14 (34\%) had grade 4 cytopenias ( $20 \%$ of cycles).

The 12 deaths reported during the study were attributed to disease progression $(n=9)$, cerebral stroke $(n=1$; 4 months after end of treatment), sepsis ( $n=1 ; 12$ months after end of treatment), and heart failure ( $n=1 ; 39$ months after end of treatment). No treatment-related deaths were recorded.

\section{Discussion}

In this phase 2 trial of 57 treatment-naive elderly patients with mantle cell lymphoma, the RBAC500 regimen was active and induced PET-negative complete response in 
$91 \%$ of patients. Progression-free survival was $81 \%$ at 2 years and $76 \%$ at the median follow-up of 35 months, without any maintenance therapy. The toxicity of RBAC500 was substantially reduced compared with results from the pilot trial of the same regimen but higher cytarabine dose ${ }^{14}$ while efficacy was comparable. The most common toxicity in our trial was grade 3-4 thrombocytopenia (52\% of cycles). Cytopenias were frequent but well managed with dose reduction, and were rarely complicated by major infective or haemorrhagic issues, making this regimen generally applicable to elderly patients (aged 80 years or younger) who are fit. However, RBAC500 was more toxic than historical controls of bendamustine-rituximab. ${ }^{5,8}$

Complete response and median progression-free survival in our study compare favourably with established first-line treatments in elderly patients with mantle cell lymphoma, including bendamustinerituximab and R-CHOP, with or without maintenance. Two phase 3 studies ${ }^{5,8}$ have investigated the role of bendamustine-rituximab in the treatment of previously untreated patients with mantle cell lymphoma and those with other histological subtypes. In the STiL study, ${ }^{5}$ median progression-free survival for the subgroup of patients with mantle cell lymphoma (median age 70 years) was 35.4 months (IQR 28.8-54.9). In the BRIGHT study, ${ }^{8}$ complete response based on PET assessment was achieved in 17 (50\%) of 34 patients with mantle cell lymphoma, but data for progression-free survival were not available. In 2016, the Nordic Lymphoma Group assessed the addition of lenalidomide to bendamustine-rituximab in 51 patients with similar characteristics as in our study population. ${ }^{21} 32$ (64\%) patients achieved complete remission, and median progression-free survival (42 months, 95\% CI 31-53) was better than that expected with bendamustinerituximab alone. However, grade 3-5 infections were reported in 21 (42\%) patients, and second primary malignancies in eight (16\%) patients. Finally, another phase 2 study $^{22}$ assessed 23 transplantation-eligible patients treated upfront with alternating bendamustinerituximab and rituximab-cytarabine. Although most (21 [91\%]) patients had low-risk or intermediate-risk MIPI and no patient had the blastoid variant, the proportion of patients achieving complete response after six treatment cycles ( $96 \%$ [22 patients]) and progressionfree survival at 13 month follow-up (96\%, 90\% CI 73-99) were similar to data in this trial and our previous study. ${ }^{14}$ This trial used the same drugs as in our trial, although in a different schedule, and had similar results to this study and our previous trial, ${ }^{14}$ thus confirming the synergism between these drugs.

In the European MCL Network's trial, ${ }^{6} 485$ patients with similar age (median 70 years, range 60-87) and risk (242 [50\%] with high-risk MIPI) to our cohort were randomly assigned to receive R-CHOP or R-FC (rituximab, fludarabine, and cyclophosphamide), and responders

\begin{tabular}{lccccc|}
\hline & Grade 0 & Grade 1 & Grade 2 & Grade 3 & Grade 4 \\
\hline Leucopenia & 0 & $91(30 \%)$ & $79(26 \%)$ & $52(17 \%)$ & $82(27 \%)$ \\
Neutropenia & 0 & $46(15 \%)$ & $109(36 \%)$ & $43(14 \%)$ & $106(35 \%)$ \\
Febrile neutropenia &. &. &. & $12(4 \%)$ & $3(1 \%)$ \\
Thrombocytopenia & 0 & $43(14 \%)$ & $103(34 \%)$ & $49(16 \%)$ & $109(36 \%)$ \\
Anaemia & $63(21 \%)$ & $73(24 \%)$ & $131(43 \%)$ & $36(12 \%)$ & $1(<1 \%)$ \\
Data are n (\%). 304 cycles were given in total. & & & & \\
\hline Table 2: Treatment cycles with haematological toxicities & & & \\
\end{tabular}

\begin{tabular}{|c|c|c|c|c|}
\hline & All grades & Grade 1-2 & Grade 3 & Grade 4 \\
\hline Nausea or vomiting & $12(21 \%)$ & $12(21 \%)$ & 0 & 0 \\
\hline Stomatitis & $3(5 \%)$ & $3(5 \%)$ & 0 & 0 \\
\hline $\begin{array}{l}\text { Infusion-related reactions } \\
\text { or tumour lysis syndrome }\end{array}$ & $12(21 \%)$ & $11(19 \%)$ & $1(2 \%)$ & 0 \\
\hline Constipation or diarrhoea & $6(11 \%)$ & $6(11 \%)$ & 0 & 0 \\
\hline Fatigue & $14(25 \%)$ & $13(23 \%)$ & $1(2 \%)$ & NA \\
\hline Documented infections & $9(16 \%)$ & $3(5 \%)$ & $4^{*}(7 \%)$ & $2 \dagger(4 \%)$ \\
\hline $\begin{array}{l}\text { Elevation of } \\
\text { glutamic-pyruvic } \\
\text { transaminase and } \\
\text { glutamic-oxaloacetic } \\
\text { transaminase or } \\
\gamma \text {-glutamyl transferase }\end{array}$ & $6(11 \%)$ & $5(9 \%)$ & $1(2 \%)$ & 0 \\
\hline Alopecia & $3(5 \%)$ & $3(5 \%)$ & 0 & 0 \\
\hline Rash or desquamation & $5(9 \%)$ & $5(9 \%)$ & 0 & 0 \\
\hline Cardiac events & $3(5 \%)$ & 0 & $2 \ddagger(4 \%)$ & $1 \S(2 \%)$ \\
\hline
\end{tabular}

Data are $n$ (\%). NA=not applicable. *One patient had Herpes zoster reactivation, two had cytomegalovirus reactivation, and one had fungal infection.

†Pseudomonas aeruginosa and Staphylococcus aureus sepsis in the course of grade 3-4 neutropenia. $¥$ One symptomatic atrial fibrillation and one chest pain without sequelae. SMyocardial infarction with cerebral ischaemia during $P$ aeruginosa sepsis.

Table 3: Non-haematological toxicity occurring in at least one patient

were randomised again to receive maintenance therapy with rituximab or interferon alfa. 81 (34\%) of 239 patients receiving $\mathrm{R}-\mathrm{CHOP}$ achieved complete response, with a median time to treatment failure of 28 months. Compared with R-CHOP, the VR-CAP regimen, which replaces vincristine in R-CHOP with bortezomib, improved the quality of response (complete response $42 \%$ vs $53 \%$ ) and significantly prolonged median progression-free survival (14.4 months vs 24.7 months). ${ }^{7}$ In the ECOG's phase 2 study, ${ }^{23} 75$ previously untreated patients with mantle cell lymphoma were given VcR-CVAD (rituximab, bortezomib, modified hyper-cyclophosphamide, doxorubicin, vincristine, and dexamethasone) induction chemotherapy. Of the 44 patients (of whom nine [22\%] had high-risk MIPI) who were ineligible for autologous stem-cell transplantation and were given rituximab maintenance instead, 31 (70\%) achieved complete response with VcRCVAD, and 3 year progression-free survival from the start of rituximab maintenance was $67 \%$ (95\% CI 52-85). In another phase 2 study, ${ }^{24}$ R-CHOP plus bortezomib, followed by bortezomib maintenance, led to a 2 year 
progression-free survival of $62 \%(95 \%$ CI $49-72)$ and a median progression-free survival of 29.5 months.

Consistent with results from the literature, ${ }^{9-11,14,24,25} \mathrm{Ki67}$ expression was the strongest predictive marker of progression-free survival, overall survival, and duration of response in our study. Patients with low Ki67 (<30\%) had a long-term progression-free survival of around $90 \%$ without maintenance therapy. The two patients with progressive disease both had elevated Ki67 ( $\geq 30 \%)$. Ki67 expression has also been reported to be a strong predictive marker of treatment response and outcome after intensive or targeted therapies, such as the combination of ibrutinib and rituximab. ${ }^{25}$ Future studies should stratify patients on the basis of this easy-to-assess variable, and newer therapeutic options for patients with elevated Ki67 are urgently needed.

In our study, most assessed patients did not have minimal residual disease (assessed by nested PCR) in the bone marrow and peripheral blood at the end of treatment. The proportion of patients with molecular response in peripheral blood at the end of treatment (79\%) was higher in our study than that assessed by realtime quantitative PCR after R-CHOP (48\%)-but similar to R-FC $(80 \%)$ or RiBVD (rituximab, bendamustine, bortezomib, and dexamethasone; 83\%)-in elderly patients with mantle cell lymphoma. ${ }^{28,29}$ In a trial of lenalidomide plus bendamustine-rituximab using nested PCR ${ }^{21}$ molecular response (19 [56\%] of 32 patients in bone marrow) was similar to that in our study.

Regarding the statistical design of this trial, our study had divergent results with respect to the targets of the primary endpoints: the proportion of patients achieving complete response was higher than expected and toxicity was higher than expected. These discordant results suggest that the overall type I error (ie, erroneously rejecting the null hypotheses) should not have been inflated under the assumption of independence between response and toxicity, even considering that deviations from this assumption have generally little effect on the Bryant and Day design. ${ }^{19}$ In our trial, toxicity was substantial but manageable. Overall, 23 patients had at least one episode of relevant toxicity, which exceeded our initial assumption (<18 of 57). Haematological toxicity, especially grade 4 thrombocytopenia (36\% of cycles), was frequent but reduced compared with results from a previous trial of R-BAC (64\% of cycles).$^{14}$ Grade 3-4 thrombocytopenia and neutropenia were reported in $70 \%$ and $74 \%$ of patients, respectively. Dose reduction was required in most (72\%) patients and was associated with reduced grade 4 cytopenias ( $20 \%$ of cycles). These frequencies are higher than those reported for R-CHOP (162 [67\%] of 242 patients had grade 3 or higher thromocytopenia and 14 [6\%] had grade 3 or higher neutropenia), ${ }^{7}$ but similar to those reported for induction regimens including bortezomib. ${ }^{7,23}$ Indeed, toxicities reported during VCR-CVAD or VR-CAP were mainly haematological, with grade 3-4 thrombocytopenia occurring in $67 \%$ of patients given VcR-CVAD and $57 \%$ of those given VR-CAP, and grade $3-4$ neutropenia occurring in $84 \%$ of those given VcR-CVAD and $85 \%$ of those given VR-CAP. By contrast, bendamustinerituximab has much lower frequency of grade 3-4 cytopenias than in the present study. ${ }^{5,8}$ In our study, nonhaematological toxicity was uncommon, and grade 3-4 alopecia was not observed.

In the past 2 years, new therapies have shown noticeable activity in previously untreated patients with mantle cell lymphoma. In a trial reported in $2015,{ }^{30}$ patients with mantle cell lymphoma treated upfront with prolonged administration of lenalidomide and rituximab $(n=38)$ had high complete response (64\%, 95\% CI 46-79), with a 2 year progression-free survival of $85 \%$ (67-94). In this study, one-third of patients had low-risk MIPI, no patient had the pleomorphic or blastoid variant, and Ki67 index was low in 26 patients (76\% of those with available data). These drugs offer new chemotherapy-free opportunities for treatment of mantle cell lymphoma and should be tested in tumours with elevated Ki67, in which chemoimmunotherapy does not offer long-term benefit.

In conclusion, our results support the use of RBAC500 in elderly patients with mantle cell lymphoma. Despite exceeding our prespecified toxicity criteria, this regimen led to high complete response, with a low frequency of severe or life-threatening adverse events. Haematological toxicity was frequent but manageable with dose reduction and supportive care. Responses were durable without the use of maintenance therapy and compared favourably with previously reported regimens, including bendamustine-rituximab, in this patient population. With several new expensive drugs on the market, RBAC500 is a regimen that is worth studying in a phase 3 trial. Further studies are needed to confirm our findings.

\section{Contributors}

$\mathrm{CV}, \mathrm{AC}, \mathrm{MM}$, and UV conceived and designed the study. All authors were involved in patient recruitment, data collection, and database assembly, and approved the final version of the report. CV, SFe, AE, SAP, ML, GC, and UV analysed and interpreted the data. CV, AC, SFe, AE, ML, GC, MM, and UV wrote the report.

\section{Declaration of interests}

We declare no competing interests.

\section{Acknowledgments}

We thank all patients who participated in this trial. We thank the Fondazione Italiana Linfomi secretary and particularly Elisa Masiera. We thank Luigia Monitillo and Paola Ghione for their help in analyses of minimal residual disease. This research was supported by Progetto di Ricerca Sanitaria Finalizzata 2009 grant (RF-2009-1469205) and 2010 grant (RF-2010-2307262); AO S Maurizio, Bolzano/Bozen, Italy; Fondi di Ricerca Locale, Università degli Studi di Torino, Italy; Fondazione Neoplasie Del Sangue, Torino, Italy; and Fondazione Cassa di Risparmio di Torino (project code 2015.1044), Torino, Italy.

\section{References}

1 Herrmann A, Hoster E, Zwingers T, et al. Improvement of overal survival in advanced stage mantle cell lymphoma. J Clin Oncol 2009; 27: $511-18$.

2 Dreyling $\mathrm{M}$, Kluin-Nelemans $\mathrm{HC}$, Beà $\mathrm{S}$, et al. Update on the molecular pathogenesis and clinical treatment of mantle cell lymphoma: report of the 11th annual conference of the European Mantle Cell Lymphoma Network. Leuk Lymphoma 2013; 54: 699-707. 
3 Lenz G, Dreyling M, Hoster E, et al. Immunochemotherapy with rituximab and cyclophosphamide, doxorubicin, vincristine, and prednisone significantly improves response and time to treatment failure, but not long-term outcome in patients with previously untreated mantle cell lymphoma: results of a prospective randomized trial of the German Low Grade Lymphoma Study Group (GLSG). J Clin Oncol 2005; 23: 1984-92.

4 Howard OM, Gribben JG, Neuberg DS, et al. Rituximab and CHOP induction therapy for newly diagnosed mantle-cell lymphoma: molecular complete responses are not predictive of progression free survival. J Clin Oncol 2002; 20: 1288-94.

5 Rummel MJ, Niederle N, Maschmeyer G, et al. Bendamustine plus rituximab versus $\mathrm{CHOP}$ plus rituximab as first-line treatment for patients with indolent and mantle-cell lymphomas: an open-label, multicentre, randomised, phase 3 non-inferiority trial. Lancet 2013; 381: 1203-10.

6 Kluin-Nelemans HC, Hoster E, Hermine O, et al. Treatment of older patients with mantle-cell lymphoma. N Engl J Med 2012; 367: 520-31

7 Robak T, Huang H, Jin J, et al. Bortezomib-based therapy for newly diagnosed mantle-cell lymphoma. N Engl J Med 2015; 372: 944-53.

8 Flinn IW, van der Jagt R, Kahl BS, et al. Randomized trial of bendamustine-rituximab or R-CHOP/R-CVP in first-line treatment of indolent NHL or MCL: the BRIGHT study. Blood 2014; 123: 2944-52.

9 Hermine O, Hoster E, Walewski J, et al. Addition of high-dose cytarabine to immunochemotherapy before autologous stem-cell transplantation in patients aged 65 years or younger with mantle cell lymphoma (MCL Younger): a randomised, open-label, phase 3 trial of the European Mantle Cell Lymphoma Network. Lancet 2016; 388: 565-75.

10 Romaguera JE, Fayad L, Rodriguez MA, et al. High rate of durable remissions after treatment of newly diagnosed aggressive mantle-cell lymphoma with rituximab plus hyper-CVAD alternating with rituximab plus high-dose methotrexate and cytarabine. J Clin Oncol 2005; 23: 7013-23.

11 Geisler CH, Kolstad A, Laurell A, et al. Long-term progression-free survival of mantle cell lymphoma after intensive front-line immunochemotherapy with in vivo-purged stem cell rescue: a nonrandomized phase 2 multicenter study by the Nordic Lymphoma Group. Blood 2008; 112: 2687-93.

12 Visco C, Castegnaro S, Chieregato K, et al. The cytotoxic effects of bendamustine in combination with cytarabine in mantle cell lymphoma. Blood Cells Mol Dis 2012; 48: 68-75.

13 Castegnaro S, Visco C, Chieregato K, et al. Cytosine arabinoside potentiates the apoptotic effect of bendamustine on several B- and T-cell leukemia/lymphoma cells and cell lines. Leuk Lymphoma 2012; 53: 2262-68.

14 Visco C, Finotto S, Zambello R, et al. Combination of rituximab, bendamustine, and cytarabine for patients with mantle-cell non-Hodgkin lymphoma ineligible for intensive regimens or autologous transplantation. J Clin Oncol 2013; 31: 1442-49.

15 Swerdlow SH, Campo E, Harris NL, et al. WHO classification of tumours of haematopoietic and lymphoid tissues. Lyon, France: IARC Press, 2008.
16 Ferrero S, Monitillo L, Mantoan B, et al. Rituximab-based pre-emptive treatment of molecular relapse in follicular and mantle cell lymphoma. Ann Hematol 2013; 92: 1503-11.

17 Voena C, Ladetto M, Astolfi M, et al. A novel nested-PCR strategy for the detection of rearranged immunoglobulin heavy-chain genes in B cell tumors. Leukemia 1997; 11: 1793-98.

18 Cheson BD, Pfistner B, Juweid ME, et al. Revised response criteria for malignant lymphoma. J Clin Oncol 2007; 25: 579-86.

19 Bryant J, Day R. Incorporating toxicity considerations into the design of two-stage phase 2 clinical trials. Biometrics 1995; 51: 1372-83.

20 Tournoux C, De Rycke Y, Médioni J, Asselain B. Methods of joint evaluation of efficacy and toxicity in phase II clinical trials. Contemp Clin Trials 2007; 28: 514-24.

21 Albertsson-Lindblad A, Kolstad A, Laurell A, et al. Lenalidomide-bendamustine-rituximab in patients older than 65 years with untreated mantle cell lymphoma. Blood 2016; 128: 1814-20.

22 Armand P, Redd R, Bsat J, et al. A phase 2 study of rituximab-bendamustine and rituximab-cytarabine for transplant-eligible patients with mantle cell lymphoma. Br J Haematol 2016; 173: 89-95.

23 Chang JE, Li H, Smith MR, et al. Phase 2 study of VcR-CVAD with maintenance rituximab for untreated mantle cell lymphoma: an Eastern Cooperative Oncology Group study (E1405). Blood 2014; 123: $1665-73$.

24 Till BG, Li H, Bernstein SH, et al. Phase II trial of R-CHOP plus bortezomib induction therapy followed by bortezomib maintenance for newly diagnosed mantle cell lymphoma: SWOG S0601. Br J Haematol 2016; 172: 208-18.

25 Hoster E, Rosenwald A, Berger F, et al. Prognostic value of Ki-67 index, cytology, and growth pattern in mantle-cell lymphoma: results from randomized trials of the European Mantle Cell Lymphoma Network. J Clin Oncol 2016; 34: 1386-94.

26 Salek D, Vesela P, Boudova L, et al. Retrospective analysis of 235 unselected patients with mantle cell lymphoma confirms prognostic relevance of Mantle Cell Lymphoma International Prognostic Index and Ki-67 in the era of rituximab: long-term data from the Czech Lymphoma Project Database. Leuk Lymphoma 2014; 55: 802-10.

27 Wang ML, Lee $\mathrm{H}$, Chuang $\mathrm{H}$, et al. Ibrutinib in combination with rituximab in relapsed or refractory mantle cell lymphoma: a single-centre, open-label, phase 2 trial. Lancet Oncol 2016; 17: 48-56.

28 Pott C, Hoster E, Delfau-Larue MH, et al. Molecular remission is an independent predictor of clinical outcome in patients with mantle cell lymphoma after combined immunochemotherapy: a European MCL intergroup study. Blood 2010; 115: 3215-23.

29 Gressin R, Callanan M, Daguindau N, et al. Frontline therapy with the RiBVD regimen elicits high clinical and molecular response rates and long PFS in elderly patients mantle cell lymphoma (MCL); final results of a prospective phase II trial by the LYSA group. Blood 2014; 124: abstr 148

30 Ruan J, Martin P, Shah B, et al. Lenalidomide plus rituximab as initial treatment for mantle-cell lymphoma. N Engl J Med 2015; 373: $1835-44$ 